\title{
An Update based Energy-Efficient Reactive Routing Protocol for Mobile Ad Hoc Networks
}

\author{
Dhirendra Kumar Sharma*, Amar Nath Patra, Chiranjeev Kumar \\ Department of Computer Science \& Engineering Indian School of Mines, Dhanbad-826 004, Jharkhand, India \\ dhirendrasharmaism@gmail.com*; amarnath.patra@gmail.com; k_chiranjeev@yahoo.co.uk
}

\begin{abstract}
Low cost energy-efficient (power based) routing protocols of mobile ad hoc networks (MANETs) increase the lifetime of static networks by using received signal strength (RSS) and battery power status (PS). They require GPS service to find the exact location of mobile nodes. The GPS devices themselves consume power because they need excessive updates to find the stationary nodes for efficient routing. To overcome this, RSS is being used as a metric, followed by, residual battery power. The recent protocols, based on these concepts, provide energy efficient routes during the route discovery phase only. Topological changes make these routes weak in due course of time. To update routes, HELLO process can be used, which however creates unnecessary overhead, delay and consumes power. Hence, these protocols do not update the routes. We propose an energy-efficient reactive routing protocol that uses the RSS and PS of mobile nodes. Proposed Link Failure Prediction (LFP) algorithm uses the link-layer feedback system to update active routes. We use ns2 for simulation of the proposed algorithm. Comparing the results of proposed scheme and existing scheme, in terms of energy consumption, link failure probability, and retransmission of packets, we observe that the proposed scheme outperforms the existing one.
\end{abstract}

Index Terms - Received Signal Strength (RSS), Battery Power Status (PS), Route Discovery, Link Failure Prediction (LFP), Route Maintenance, Routing protocols

\section{INTRODUCTION}

A mobile ad hoc network (MANET) is a frequently deployed wireless network in which mobile nodes themselves act as routers to exchange resources [14, 20]. The main challenge of MANET is continuation of routing in dynamic environment. Many routing protocols have been developed and are categorized as proactive, reactive and hybrid protocols [13]. In MANET, mobility causes a serious problem of link failure in active routes. An active route is maintained by link connectivity algorithm ([27]), that requires high transmission power and periodic route updates. Here, one of the solutions is to use a routing protocol based on RSS and node stability.

A signal stability-based adaptive (SSA) routing protocol [17] has been proposed to find the destination node for which it requires high signal strength and exact location of mobile nodes through GPS service. An enhanced-SSA $\left(\mathrm{SSA}^{+}\right)$[25] based routing protocol has an improved performance in throughput and reduced overhead than SSA as it uses high density and low mobility factor. A route established on the basis of high RSS does not guarantee in an increase of its own lifetime and the lifetime of the comprising nodes. In a static network, battery power status (PS) is used to estimate the node lifetime as well as to calculate the link lifetime on the basis of energy drain rate [6]. A link lifetime is characterized by signal strength, energy consumption rate and energy capacity. Link stability based models have been proposed to increase the lifetime of routes by using RSS [18].

Many power-aware source routing protocols have been proposed to find multiple routes between a pair of source and destination nodes [3]. In these routing protocols, RSS and battery power thresholds are used for selection of nodes during the route discovery process. The total information of the route, contained in the header of each data packet, is stored in route cache of a mobile node, which is used to compute the average power consumption of the route. The lifetime of routes is updated only by link-layer measurement (HELLO process) or link-layer feedback mechanism (through ACK packets). Mobility makes the update process difficult.

Further, EDNR protocol [26], also addresses the battery power exhaustion of mobile node. This protocol considers energy and distance as metric for prediction of link lifetime and node lifetime. It selects the least dynamic route for effective delivery of packets. For implementation as a multipath routing protocol, it uses the route-caching scheme of dynamic source routing (DSR) protocol [5]. It calculates average power consumption on available routes and then selects the minimum power consumption route. It uses the node lifetime (NLT) and link lifetime (LLT) algorithm. In this paper, authors consider two metrics: energy cost and mobility factor for long route lifetime estimation. Here, a dynamic network causes link failure and continuous updates, thereby increasing overhead.

In [29], authors state that the exhaustion of node battery power is due to high traffic load. Here, an energy mean (EM) algorithm has been proposed to increase the lifetime of network. This algorithm controls the over exhaustion of node battery power by finding low cost routes and load-balancing approach. It distributes the heavy traffic among multiple routes. These multiple 
routes stop reroute discovery process. Hence, its performance reduces in dynamic network because random mobility of nodes causes link failure on multiple routes. Thus, lifetime of link and residual battery power are only suitable for nodes that are stationary. Moreover, EM-AODV [30] increases the lifetime of network by reducing the unnecessary control packets. A cross-layer approach enhances the performance of energy aware AODV routing protocol because of the extra field for RSS added in control packets [31].

The main problem of energy aware routing protocols is battery power drainage, which degrades the performance of mobile node in terms of lifetime. In a network, power consumption increases due to reroute discovery, route update, large size of packets, and minimum hop count routing. So, low energy cost routing protocols are required to increase the performance of mobile node. Our proposed methods are inspired by these problems. We are considering RSS and PS as metric to improve the performance of routing protocol in dynamic networks. In this paper we are proposing route discovery, route maintenance and link failure prediction (LFP) algorithms to improve the performance. Proposed route discovery process uses RSS and PS to select the efficient node for routing. We are adding two extra pieces of information in controls packets, modified route request (RREQ) and route reply (RREP) packets. We have considered hop-byhop routing approach, so AODV routing protocol is used for implementation.

The major function of LFP algorithm is to monitor the active link by received packets. We are proposing route maintenance process to repair the broken link. We assume that during the mobility, link update (HELLO) process updates the status of single hop neighbor nodes with RSS and PS. In our route maintenance process, upstream node checks the position of link break and the node density for a faster route repair. Using NS2, we analyze that proposed technique gives improved result comparing to the existing method [29].

This paper is structured as follows. Basic overview of routing protocols is presented in section 2. Section 3 describes the proposed methods that cover route discovery, link failure prediction, and route maintenance process. Section 4 shows the performance evaluation of proposed and existing methods. Finally, we conclude the approach of this paper in section 5 .

\section{RELATED WORKS}

Recently, many routing protocols have been developed and categorized as proactive, reactive and hybrid protocols. The need of routing protocols is to provide effective delivery of packets with minimum energy consumption and end-to-end delay. We discuss some of the most popular energy consumption models and highlight their main issues, later in the following subsections.

In $[15,23]$, authors discuss the importance of proactive power aware routing protocols. It works well when all mobile nodes have equal amount of power in static mode.
In dynamic topology, Bellman-Ford and Dijkstra's algorithms based routing protocols continuously update the routing table. Here, update of routing table increases the overhead and power consumption. Node density also affects the performance of routing protocols [28]. In [2], node density based scheme is proposed to repair broken link by finding the position of link break and node density along the active route. This technique reduces unnecessary overhead and delay in the network.

Huge update in link state routing protocol is overcome by reactive routing protocols, it uses two phases; route discovery and route maintenance. Route discovery process is used to setup a path between source destination pair. Some of the most prevalent on-demand routing protocols are Ad-Hoc On-Demand Distance Vector (AODV) [1] and Dynamic source routing (DSR) [5]. They use intermediate nodes for forwarding the data packets. All intermediate nodes on active route have routing table, (alternate routing table, ART) and neighbor table. In the case of link failure, an upstream node sends a route error (RERR) message to source node and starts the local repair process or global repair process. After receiving RERR message, the source node stops the data transfer and starts re-route discovery. As compared to high-density network the low-density network has low probability of route repair. AODV-ABR [24] uses backup routes for route maintenance and sends packets through alternate paths. AODV-ABR enhances the throughput by minimizing the number of attempts of re-route discovery, but it is not suitable for dynamic network. Operation of alternate paths works efficiently if adjacent nodes exist at broken link. This protocol works well and gives better performance for static networks, however in case of mobility, its performance decreases. Its performance depends only on the density of adjacent nodes.

In this paper, we are using the overhearing process (promiscuous mode in ns2). The process of receiving the packets by nodes, not addressed to them, is called overhearing. The overhearing process does not increase overhead in the network because no additional packets are transmitted in response, but it consumes energy as discussed in [7]. We know that overhearing happens only in single hop so; transmission zone decides the power consumption of a node in active session. Controlledoverhearing reduces power consumption in networks. A conditional overhearing technique, RandomCast [21], considers different cases for overhearing at MAC layer. It decides when and what to overhear in the network. Our proposed scheme uses multihop architecture [8, 12, 19] for routing of data packets. In a network, high transmission power increases the interference region and battery power consumption, which results in low link lifetime. Hence, low transmission power based routing protocols reduce the interference, collision, overhearing and other factors that degrade the performance.

\section{A. Power based routing protocol}

In power aware source routing [13], header of each data packet contains the total information of route and stores it in route cache of mobile node. Power based 
routing protocol computes the average minimum power consumption of a route. This type of routing protocols uses only the routing cache of intermediate nodes to store the average power consumption cost. Periodic update process is used to increase the lifetime of route. Finally power based source routing protocols work well for stable networks. Routing protocols provide the efficient way to route data packets. One of the most important parameter is lifetime of a route. Battery-power is always an important issue in MANET. It decides the link lifetime and route lifetime. Network stability increases the lifetime of a route very easily. In power based routing protocols, RSS is the main key for selection of stable paths. Many routing protocols use RSS as a key factor. Shortest hop routing is useful for short interval service because it uses maximum transmission power and utilizes maximum bandwidth. Here, multihop routing reduces the use of high transmission power and drainage of battery power. A survey on energy aware routing protocols is discussed in [10] with various algorithms that describe RSS and battery power as main parameters.

Many routing protocols have been developed to increase the lifetime of a route. One of the classifications is multipath routing protocols, in which a source node has more than one routes to reach a destination node. The most important factor is battery power that affects the route lifetime and thereby, the network lifetime. In MANET, mobility of nodes acts as a catalyst to increase the power consumption rate; high mobility factor causes the maximum update and link break problem in multipath routing protocols. A bandwidth-based power aware routing [4] is proposed to avoid the use of GPS service. It uses bandwidth and link lifetime for selection of paths. Here, remaining power is used for prediction of link lifetime. Moreover, minimum transmission power consumption routing (MTPCR) [3], reduces the power consumption during the transmission of data packets. MTPCR finds a high-bandwidth path to decrease power consumption. This protocol decreases the path breakage during the mobility because it selects the most stable path. In our proposal we are considering a dynamic network in which except source and destination all intermediates nodes are free to move. Proposed route maintenance process starts by finding node density and position of link break. LFP algorithm helps to find weak nodes to avoid packet drops and retransmission of data packets up to threshold value. We are using HELLO process to update the neighbor table with RSS and power status. In simulation we reduce the HELLO interval time for high mobility.

Energy aware on demand routing protocols use the set of high residual energy nodes for routing of packets. This method shows low overhead for minimum energy consumption. This type of protocol uses multipath source routing because during the link break, upstream node sends RERR message to source node and starts routing through next path otherwise, restarts route discovery process. This protocol uses link-layer feedback mechanism to receive ACK packet for link break confirmation [32]. An energy-efficient routing protocol
PEER [8] is proposed to find the low cost energyefficient path by route discovery process. It reduces the maximum overhead and path setup delay during the route discovery. Route maintenance process is used to find the low cost path without using any link update process.

Mobility models are used to estimate the cost of link lifetime [9, 26]. In exploring dynamic nature routing (EDNR) [26], route prediction algorithm works on the basis of least dynamic route for longest data forwarding. EDNR uses the node lifetime and link lifetime for the prediction of lifetime of a route. In route discovery process it uses the route request (RREQ) and route reply (RREP) which contain the received signal strength and receive time in the header field. A mobile node stores this information in its local memory. We are implementing our proposed method with the help of AODV routing protocol, for this we are proposing two algorithms for route request and route reply processing.

\section{PROPOSED METHOD}

In this section we discuss the proposed methodology for energy conservation. Previously, we discussed the power related issues. In this part, we are describing route discovery process with two algorithms, route request processing, and route reply processing. Later, we propose a link-failure prediction algorithm to detect weak link and control the retransmission (10, [AODV]) and reroute discovery process. Route maintenance process works by finding node density and position of link break.

\section{A. Route discovery process}

As we know that route discovery process is used to search the destination node, we are finding low energy cost route by RSS and PS. In RREQ processing, RSS and $P S$ are compared to their threshold values. This mechanism avoids weak links and updates the routing table. Similarly, when destination node receives the first RREQ packet, it waits for a fixed time ( $3 \times$ Node traversal time [29]) to receive more RREQ packets. Destination node unicasts the RREP packets for all received RREQ packets.

During the route discovery process, Algorithm-1 and Algorithm-2 add two new entries RSS and PS in routing table. Further, we are proposing link failure prediction (LFP) technique to avoid the packet drops and retransmissions. In LFP algorithm, we are using RSS and $P S$ threshold values for finding weak link and low battery node.

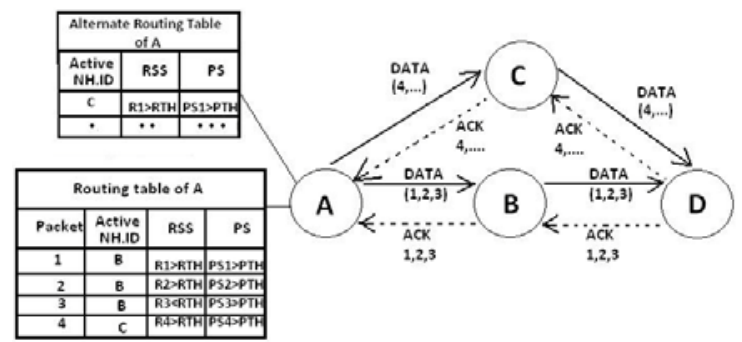

Figure. 1. Data packet transfer from source A to destination node $\mathrm{D}$ 
Example: As shown in fig. 1, source node A starts the route discovery process to find the destination node $\mathrm{D}$. Node A broadcasts modified RREQ with two new field $R S S$ and $P S$. When neighbor nodes (C, B) receive the RREQ packets, they check the RSS and PS values against the corresponding threshold values. After this they start to forward the packets to their neighbor nodes. The destination node $\mathrm{D}$ receives the RREQ packets and waits for fixed time [29] to receive more than one RREQ packets. After the timer expiry, destination D starts to reply by sending RREP packet(s) to the node(s) from which it received RREQ packets (as discussed in Algorithm 2). Each node updates its routing table with the help of RREP packets and these packets contain the latest RSS and PS values to update the routing table. It selects a route on the basis of RSS and PS. Source node A sends the RREP ACK packet (as discussed in RFC 3561) to destination node $\mathrm{D}$ for confirmation of the route, when multiple RREP packets are received.

ALGORITHM 1: On receiving RREQ packet by a node Input: RREQ packet $\mathrm{p}$

1. hdr_rreq *rq = HDR_RREQ(p); //extract the AODV route request header

2. if $\mathrm{rq}->\mathrm{src}==\mathrm{id} \| \mathrm{id} \_$lookup(rq->src) //if the receiving mobile node is itself the source or has already received $a$ duplicate then discard it

then

2.1 discard(p);

2.2 exit();

// establishing reverse route

3. rt_entry $*$ ent $=$ rtable_lookup $(\mathrm{rq}->\mathrm{src})$;

4. if ent $==0$ //if entry does not exist in the route table create an entry for the reverse route. then

4.1 ent $=$ rtable_add(rq->src);

//updating the entry on the basis of rss and ps values

5. if (rq- $>$ rss $>$ ent- $>$ rss $\& \&$ rq- $>$ ps $==$ ent $->$ ps) $\|$

$(\mathrm{rq}->\mathrm{rss}==$ ent- $>$ rss $\& \&$ rq- $>$ ps $>$ ent- $>$ ps) $\|$

$(\mathrm{rq}->\mathrm{rss}>$ ent $->$ rss $\& \&$ rq- $>$ ps $>$ ent- $>$ ps) then

5.1 if rq- $>$ rss $>$ rss_TH $\& \&$ rq- $>$ ps $>$ ps_TH then

5.1.1 rtable_update(ent);

//checking whether an RREP can be sent

6. rt_entry rt = rtable.rt_lookup(rq-> dst);

7. if $\mathrm{rq}->\mathrm{dst}==$ id $\|$ ( $\mathrm{rt} \& \& \mathrm{rt}->$ hops $!=\infty$ ) // if the receiving mobile node is the destination or has a fresh route

then

7.1 sendReply();

7.2 exit();

else

7.3 forward(p);

8. exit;

ALGORITHM 2: On receiving RREP packet Input: RREP packet $\mathrm{p}$

1. hdr_rrep *rp $=$ HDR_RREP(p);//extract the AODV route reply header
2. rt_entry $*$ ent $=$ rtable_lookup(rp->dst)

3. if ent $==0$ //if entry does not exist in the route table then

//then create an entry for the forward route

3.1 ent = rtable_add(rq->dst);

//updating the entry on the basis of rss and ps values

4. if (rp->rss $>$ ent $->$ rss \&\& rp->ps $==$ ent $->$ ps) $\|$

$(\mathrm{rp}->\mathrm{rss}==$ ent- $>$ rss \&\& rp- $>$ ps $>$ ent- $>$ ps) $\|$

(rp->rss $>$ ent- $>$ rss $\& \&$ rp- $>$ ps $>$ ent- $>$ ps) then

$$
\begin{aligned}
& 4.1 \text { if } \mathrm{rp}->\mathrm{rss}>\mathrm{rss} \_\mathrm{TH} \& \& \text { rp- }>\mathrm{ps}>\mathrm{ps} \_\mathrm{TH} \\
& \text { then } \\
& 4.1 .1 \text { rtable_update(ent); }
\end{aligned}
$$

5. if dest_addr $==$ id //if source receives the RREP then it discards it

then

5.1 discard(p);

else

5.2 rt_entry *ety = rt_lookup(dest_addr);

5.3 if ety //if entry to destination of p exists in routing table then forward the packet otherwise discard it then

5.4 else

5.3.1 forward(p);

6. exit;

5.4.1 discard(p);

\section{B. Link failure prediction}

Link-failure prediction algorithm is useful to avoid unnecessary packet drop. We assume that when link failure is detected by weak RSS, and low PS of active neighbor mobile nodes, then active upstream node selects new path from ART, which is periodically updated by HELLO process. Before selection of path, it confirms that neighbor node retains maximum amount of power. Here, length of route may increase, though nominally, by use of alternate paths for successful delivery of packets.

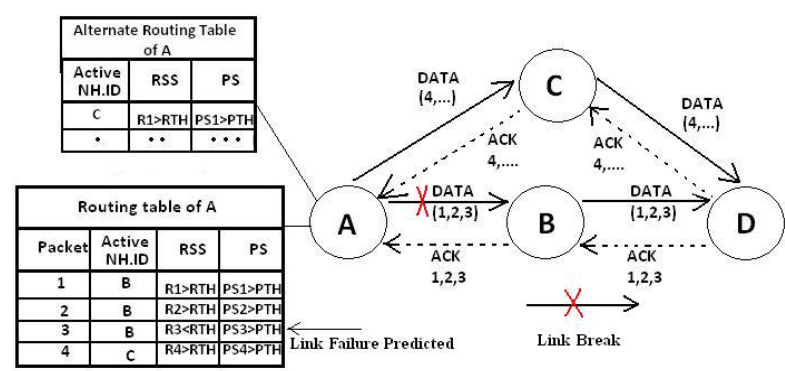

Figure. 2. Link-failure prediction process

\section{Example:}

In Fig. 2, the upstream node A discovers the weak link on active route by the LFP algorithm while receiving packets. The ACK packet sent by node $B$ to node $A$ contains its RSS and PS. Node A checks the received RSS and PS values and compares them with the corresponding threshold values (TH). If RSS and PS values of node $\mathrm{B}$ are less than $\mathrm{TH}$ values then node $\mathrm{A}$ 
predicts that the connection to node B has become weak. Now node A selects the entry of node $C$ from its alternate routing table. There are two cases. The first case is of a static network where there is no need of periodic HELLO processes. The second case is of dynamic network where the nodes maintain the link connectivity through the periodic HELLO process.

Interruption on link (due to topological changes) is called link failure. In this paper we are considering two metrics RSS and PS for link lifetime. The lifetime of links is affected by mobility, traffic rate, size of packet and low battery power. In normal routing protocol, link lifetime is increased by link update (or HELLO process). Availability of link shows that mobile nodes reside inside the coverage area. Unified link failure and recovery architecture (ulfra) performs the failure detection by link layer measurement and link layer acknowledgment [33]. Our proposed link failure prediction algorithm predicts the link failure while receiving any type of packet and monitors the link (active route) with minimum use of HELLO process.

ALGORITHM 3: Link failure prediction Input: Packet p (a reply packet to either control or link layer packet)

1. hdr *rpy $=\operatorname{HDR}(\mathrm{p})$; / /extract header of packet $p$

2. if rpy->rss < rss_TH //if rss value is less than the threshold then

2.1 if num_trans $>$ LP_TH

//if number of re-transmissions (10) is greater than threshold

then

2.1.1 if dist $>$ d_TH

//if (calculated) distance is greater than threshold then

2.1.1.1 if art_lookup(dst)

//if alternate path exists in alternate routing table then

\subsubsection{2 else}

2.1.1.1.1 perform route update

2.1.1.1.2 perform route repair

3. exit;

\section{Route maintenance process}

In MANET, during the topological changes, on demand routing protocols repair the broken link by route maintenance process. Many recent routing protocols [2, $22,24]$ have been developed to repair the broken link in different scenarios. In our proposed method, we are using AODV routing protocol for the implementation. We modify the route request and route reply packets as discussed in Algorithms 1 and 2. A route maintenance process starts when alternate paths are not available and considers three cases on the basis of position of link break:

1. Nearest to destination node,
2. Middle of route, and

3. Nearest to source node

In Fig. 3 upstream node A does not has alternate paths in ART and then starts the route maintenance process. In the first case, route maintenance starts when the position of link break is nearest to destination node. In the second case, it finds the density of neighbor nodes and continues the route maintenance process as discussed in [24]. In last case, upstream node sends RERR message to source node, which starts reroute discovery.

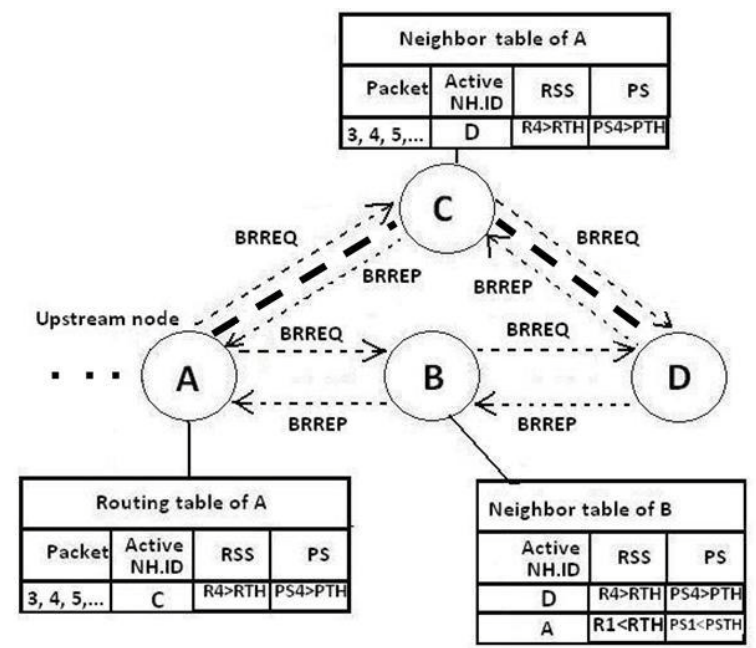

Figure. 3. Data transfer after route maintenance

Procedure for Route maintenance (check the ART)

1. If alternate paths not available then

1.1 check position of link break

1.2 if position of link break nearest to destination node then

1.2.1 start route maintenance process //as discussed in [24]

1.3 else if position of link break in middle of a route

then

1.3.1 check neighbor node density

1.3.2 if neighbor node density sufficient then

1.3.2.1 start route maintenance process

1.3.3 else send route error (RERR) message to source node

1.4 else

1.4.1 source node starts reroute discovery //as discussed in section 3.1

2. else send data packets through alternate paths.

3. exit.

\section{PERFORMANCE EVALUATION}

In performance evaluation following parameters are used and define as in table-I. 
Table: I. Nomenclature of performance analysis

\begin{tabular}{|c|c|}
\hline Notations & Description \\
\hline$H$ & Average number of hops of a route \\
\hline$M f$ & Mobility factor \\
\hline$P C_{T X}$ & $\begin{array}{l}\text { Power consumption during } \\
\text { transmission }\end{array}$ \\
\hline$P C_{R X}$ & Power consumption during receiving \\
\hline$P C_{A C K}$ & $\begin{array}{l}\text { Power consumption by receiving and } \\
\text { transmission of ACK packets }\end{array}$ \\
\hline$P C_{O}$ & $\begin{array}{l}\text { Power consumption by overhearing } \\
\text { process }\end{array}$ \\
\hline$P C_{\text {HELLO }}$ & $\begin{array}{l}\text { Power consumption during HELLO } \\
\text { process }\end{array}$ \\
\hline$P C_{\text {INITIAL RD }}$ & $\begin{array}{l}\text { Power consumption during initial } \\
\text { route discovery process }\end{array}$ \\
\hline$P P_{L F}$ & Probability of link failure \\
\hline $\begin{array}{l}\text { PC ROUTE } \\
\text { MAINTENANCE }\end{array}$ & $\begin{array}{l}\text { Power consumption during route } \\
\text { maintenance process }\end{array}$ \\
\hline$P C_{\text {ALTERNATE LINKS }}$ & $\begin{array}{l}\text { Power consumption by sending data } \\
\text { packets through alternate links }\end{array}$ \\
\hline PC & $\begin{array}{l}\text { Power consumption by sending data } \\
\text { packets through multipath }\end{array}$ \\
\hline$N$ & Number of routes from source node \\
\hline
\end{tabular}

In this section we discuss the effect of mobility on link lifetime and node lifetime. In our simulation, there are two cases for mobility (as shown in Fig. 4 (a, b)) -

1. Slow motion: neighbor nodes remain inside coverage area, and

2. High-speed motion: neighbor nodes cross the coverage area

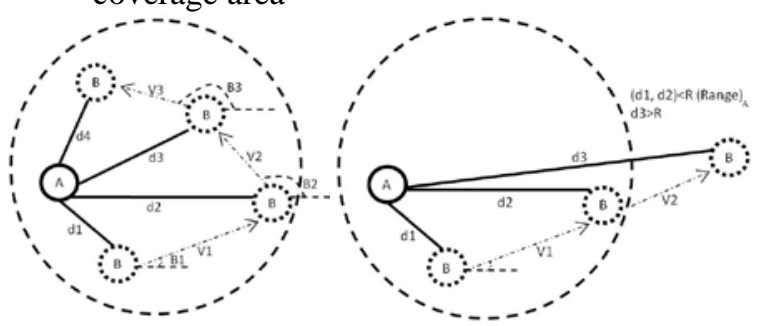

Figure. 4 (a). movement of nodes with slow speed (inside the coverage area), Fig. 4(b). high-speed mobility (crossing the coverage area) in small duration

A node crosses the coverage area on the basis of its speed and degree of mobility. The node B in Fig. 4 (a) is moving slowly with a fixed angle. In this case there is a very high probability that it remains inside the coverage area of node A. Existing link availability model [16] calculates the predicted lifetime of link without considering energy. Here speed is the prime factor for deciding the existence of mobile node inside the coverage area. Mobility of node causes weak RSS and requires high transmission power for link update to maintain the connectivity, but this process consumes maximum energy. Moreover, this connectivity maintenance approach is not suitable for node lifetime. Threshold power is the minimum power required to transmit or receive data packets.
In [11], the authors proposed an energy consumption model:

$$
P C=m^{*} \text { size of packet }+b
$$

RSS and PS of neighbor node help to predict link failure. The lifetime of link depends on lifetime of node. We are using the lifetime of node by [6] equation,

$$
T_{i}(q)=P S_{i} /\left(\sum_{j \in S} P S_{i j} \sum_{c \in C} q_{i j}^{(c)}\right)
$$

A link's lifetime is increased by low data transfer rate, which is useful for multi-hop architecture. In general, a routing protocol assumes that if distance between nodes increases then high transmission power is required for maintaining the connectivity. In our proposed model, we consider a node lifetime, $T_{i}(q)$ and $i^{\text {th }}$ link of a route, $l_{i}$. Suppose the lifetime of link is $X_{l_{i}}, i=1,2, \ldots, k$, which is a set of independent distributed (iid) exponential random variables with mean $\boldsymbol{I}$. In our proposed technique, we assume that lifetime of link increases due to high node lifetime and low mobility factor. Average lifetime of link is computed as,

Lifetime of $\operatorname{link}\left(X_{l_{i}}\right)=\left(\max \left(T_{i}(q)\right)\right.$, mobility factor $)$

We know that a route is formed by a set of selected nodes, known as active nodes. AODV is based on hopby-hop routing and all active nodes have routing table to forward the data packets. We consider the low cost energy consumption route, which is monitored by LFP algorithm. Average lifetime of a route is calculated as:

Lifetime of route $=\max \left(\frac{1}{h} \sum_{h}(\right.$ Lifetime of link $\left.)\right)+\sum L_{\text {alternate lifanks }}^{\text {lifetime }}$

Overall power consumption is given by,

Total Power Consumption $=$

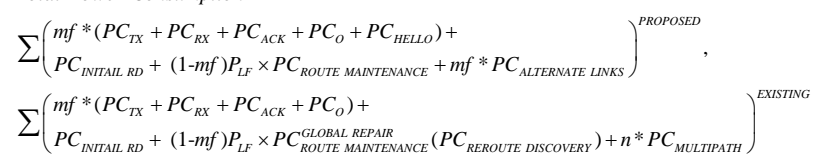

Use of ART and node density increases the connectivity between nodes and is helpful for increasing the lifetime of a route. In this paper, the actual lifetime of route depends on low cost energy consumption. Initially, all nodes have equal battery capacity, which is consumed by various processes: routing of large data packets, high traffic, overhearing process, and reroute discovery process. Our link failure prediction method reduces the reroute discovery by exploring the utility of ART. We update the ART by HELLO process (HELLO interval = $500 \mathrm{~ms}$ ) to exchange the RSS and PS information. 
Table-II, Power consumption measurement [11]

\begin{tabular}{|l|l|}
\hline $\begin{array}{l}\text { Point-to-point } \\
\text { send }\end{array}$ & $\begin{array}{l}1.9 \times \text { size of packet }(\mu W \text {-sec/byte })+454 \\
(\mu W \text {-sec })\end{array}$ \\
\hline Broadcast send & $\begin{array}{l}1.9 \times \text { size of packet }(\mu W \text {-sec/byte })+266 \\
(\mu W \text {-sec })\end{array}$ \\
\hline $\begin{array}{l}\text { Point-to-point } \\
\text { receive }\end{array}$ & $\begin{array}{l}0.5 \times \text { size of packet }(\mu W \text {-sec/byte })+356 \\
(\mu W \text {-sec })\end{array}$ \\
\hline $\begin{array}{l}\text { Broadcast } \\
\text { receive }\end{array}$ & $\begin{array}{l}0.5 \times \text { size of packet }(\mu W \text {-sec/byte })+56 \\
(\mu W \text {-sec })\end{array}$ \\
\hline $\begin{array}{l}\text { Promiscuous } \\
\text { receive }\end{array}$ & $\begin{array}{l}\text { Non-destination } n \in S, D \\
0.39 \times \text { size of packet }(\mu W \text {-sec/byte })+140 \\
(\mu W \text {-sec })\end{array}$ \\
\hline Discard & $\begin{array}{l}-0.61 \times \text { size of packet }(\mu W \text {-sec/byte })+70 \\
(\mu W \text {-sec })\end{array}$ \\
\hline $\begin{array}{l}\text { Promiscuous } \\
\text { receive }\end{array}$ & $\begin{array}{l}0.54 \times \text { size of packet }(\mu W \text {-sec/byte })+66 \\
(\mu W \text {-sec })\end{array}$ \\
\hline Discard & $\begin{array}{l}-0.58 \times \text { size of packet }(\mu W \text {-sec/byte })+24 \\
(\mu W \text {-sec })\end{array}$ \\
\hline Promiscuous & $\begin{array}{l}\text { Non-destination } n \notin S, n \in D \\
0.0 \times \text { size of packet }(\mu W \text {-sec/byte })+63 \\
(\mu W \text {-sec })\end{array}$ \\
\hline receive & $\begin{array}{l}0 \times \text { size of packet }(\mu W \text {-sec/byte })+56(\mu W- \\
\text { sec })\end{array}$ \\
\hline Discard & $843 m W$ \\
\hline Idle (ad hoc)
\end{tabular}

\section{A. Simulation environment}

The performance of proposed method evaluated in ns2 and following parameters are used during the simulation. We use network simulator (ns2.34) for our simulations. AODV routing protocol is used to implement the proposed algorithms, as mentioned earlier. It is observed that our proposed algorithms give better result than the existing routing protocol ([29]). The existing routing protocol computes the average energy consumption on active routes in high stability. We check the effect of mobility on the performance of enhanced power based AODV routing protocol. A mobile node first starts to move inside the coverage area.

We observe that slow mobility does not have any major impact on the performance of routing protocol. We run more than one CBR applications and compute the energy consumption. In existing AODV routing, number of retransmission attempts is 10 as discussed in [1]. In our proposed method, a node predicts weak link before its failure and sends the data packets through alternate paths. We evaluate the performance of our proposed method comparing to the AODV routing protocol. We are reducing the energy consumption by using multihop architecture and minimum transmission power. We know that shortest path is useful for short time communication with high data transfer rate, but this methodology is not useful for increasing the route lifetime. In our simulation we set the transmission power of mobile node to 50meters. We set the maximum HELLO interval, $1500 \mathrm{~ms}$ and minimum, 500ms. We check the effect of overhearing by implementing promiscuous mode in ns2.
Table: III Simulation parameters

\begin{tabular}{|l|l|}
\hline Parameter & Value \\
\hline Simulation time & $600 \mathrm{~s}$ \\
\hline Topology Size & $500 \times 500 \mathrm{~m}^{2}$ \\
\hline $\begin{array}{l}\text { Number of mobile } \\
\text { nodes }\end{array}$ & 50 \\
\hline MAC Type & MAC 802.11 \\
\hline $\begin{array}{l}\text { Radio propagation } \\
\text { model }\end{array}$ & Two ray ground \\
\hline Range & $50 \mathrm{~m}$ \\
\hline Energy model & Energy model \\
\hline Size of packet & 512 Bytes \\
\hline Initial Energy & $150 \mathrm{~J}$ \\
\hline $\mathrm{T}_{\mathrm{x}}$ Power & $0.281838 \mathrm{Watt}$ \\
\hline $\mathrm{R}_{\mathrm{x}}$ Threshold & $7.69113 \times 10^{-8} \mathrm{Watt}$ \\
\hline Traffic type & CBR \\
\hline CBR rate & $1 \mathrm{Mbps}$ \\
\hline Promiscuous mode & Enable \\
\hline Hello interval & $1000 \mathrm{~ms}$ \\
\hline Data size & $5 \mathrm{MB}$ \\
\hline Speed & 1 to $10 \mathrm{~m} / \mathrm{sec}$ \\
\hline
\end{tabular}

\section{B. Result and discussion}

The first result, Fig. 6, illustrates the energy consumption with respect to number of packets. We notice that our proposed method gives improvement of $58.921 \%$. Our second result as shown in Fig. 7 gives an improvement of $28.69 \%$ and shows energy consumption with respect to packet flow rate. Here, existing method uses the link-layer feedback mechanism and goes to maximum attempt for successful delivery, so it consumes excess energy. We are using HELLO process to find the node density for route maintenance. Proposed link failure prediction algorithm is used to monitor the routing table with the help of RSS and PS. Fig. 8 shows the result when link failure probability becomes high. It increases when all mobile nodes move with high speed. Our proposed method achieves 26.9\% improvement. Finally, Fig. 9, shows the analysis of different ranges of mobile nodes and transmission time to complete a simulation.

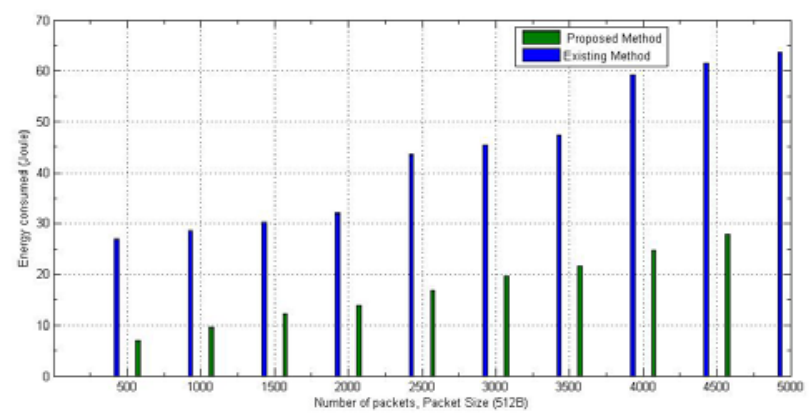

Figure. 6. Energy consumption with respect to number of packets 


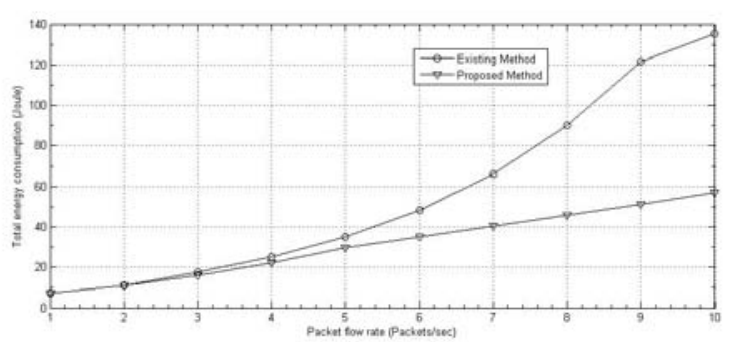

Figure. 7. Energy consumption vs. packet rate

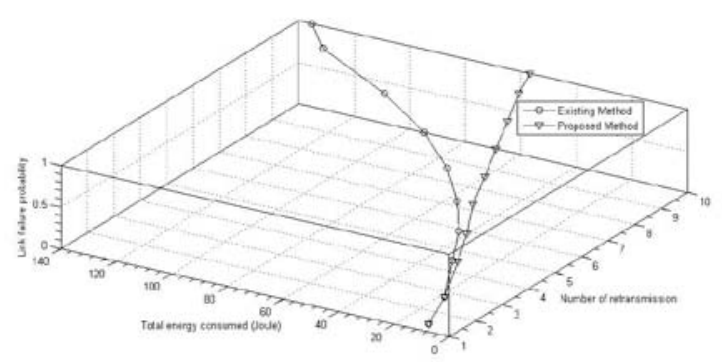

Figure. 8. Energy consumption vs. link failure probability in mobility

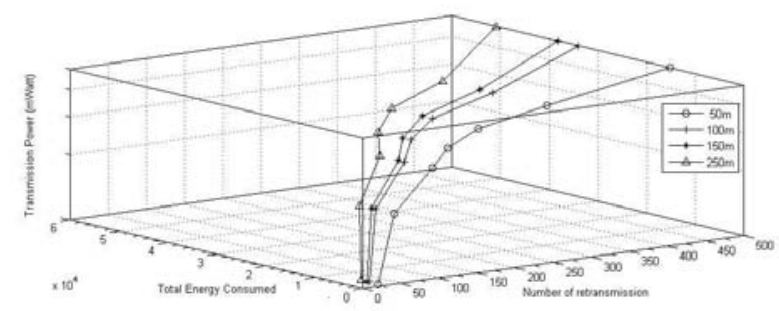

Figure. 9. Total energy consumption (High mobility)

\section{CONCLUSIONS}

In on-demand routing protocols, a set of links (route) is formed by route discovery process whose proper maintenance increases the lifetime. In our proposed technique, periodic update and overhearing process consume less energy, because the periodic update (HELLO process) starts only when there is any mobility. We are using hello intervals, $1500 \mathrm{~ms}$ and $500 \mathrm{~ms}$, for static and dynamic networks respectively, to update the entries of the routing table (and ART). The high-speed movement of nodes is the major weakness of RSS. It increases the distance between nodes and requires high transmission power to cover it. This in turn rapidly discharges the battery power (PS). Another factor of high-energy consumption is the retransmission attempts for successful delivery. Our proposal is inspired by energy aware routing protocols. In this paper, we have proposed three algorithms: route discovery (RREQ processing, RREP processing), link-failure prediction and route maintenance. Our proposed route discovery is used to find the low cost energy efficient route on the basis of RSS and PS. Link failure prediction algorithm helps to monitor the active route which avoids the retransmission of packets through weak link. It works by the means of received packets which contain, latest RSS and PS. After the link failure process, we start the route maintenance as discussed in route maintenance algorithm. The objective of our proposed method is to maintain the continuity of routing without consuming unnecessary energy. Our proposed method does not use the load balancing approach for load distribution because multipath routes are broken by random mobility of mobile nodes. Our results show that proposed method reduces the power consumption during the topological changes. We reduce the receive threshold of nodes to control the overhearing process. In our simulation only active nodes start the HELLO process. We establish low cost energy routes using the proposed route discovery process and maintain the broken link by enhanced route maintenance process.

\section{REFERENCES}

[1] C. E. Perkins, et al., 2003. Ad hoc On-Demand Distance Vector (AODV) Routing. IETF RFC 3561, pp. 1-35.

[2] C. W. Yu, et al., 2007. A low overhead route repairing mechanism for mobile ad hoc networks. Computer Communications, vol. 30 (5), pp. 11521163.

[3] C.-W. Chen, C.-Chi Weng, 2012. A power efficiency routing and maintenance protocol in wireless multihop networks. The Journal of Systems and Software, vol. 85 (1), pp. 62-76.

[4] C.-W. Chen, et al., 2010. Signal strength routing for power saving in mobile ad hoc networks. The Journal of Systems and Software, vol. 83 (8), pp. 1373-1386.

[5] D. B. Johnson, D. A. Maltz, 1996. Dynamic source routing in ad hoc wireless networks. In: Proceeding of Computer Communication Review- Proceeding of SIGCOMM.

[6] D.-Y. Hwang, et al., 2006. EASR: An energy aware source routing with disjoint multipath selection for energy-efficient multihop wireless ad hoc networks. In: Proceeding of International Conference Wireless Communications, Networking and Mobile Computing, vol. 3976, pp. 41-50.

[7] J. Gomez, A. T. Campbell, 2004. A case for variablerange transmission power control in wireless multihop networks. In: Proceedings of IEEE Computer and Communications Societies (INFOCOM), vol. 2, pp.1425-1436.

[8] J. Zhu, X. Wang, 2011. Model and protocol for energy-efficient routing over mobile ad hoc networks. IEEE Transactions on Mobile Computing, vol. 10, (11), pp. 1546-1557.

[9] Jianzhen Sun, et al., 2010. Link stability based routing in mobile ad hoc networks. In: Proceedings of IEEE international conference on industrial electronics and applications, pp. 1821-1825.

[10] S. Mahfoudh, P. Minet, 2008. Survey of energy efficient strategies in wireless ad hoc and sensor networks. In: Proceedings of $7^{\text {th }}$ International conference on networking (ICN'08), Cancun, Mexico, pp. 1-7. 
[11]L. M. Feeney, 2001. Investigating the energy consumption of a wireless network interface in an ad hoc networking environment. In: Proceedings of IEEE Computer and Communications Societies (INFOCOM), vol. 3, pp. 1548-1557.

[12]L. T. Dung, et al., 2012. An analytical performance evaluation of multi-hop paths in mobile ad-hoc wireless networks. In: Proceedings of International Conference on Ubiquitous and Future Networks (ICUFN), pp. 58-62.

[13] M. Abolhasan, et al., 2004. A review of routing protocols for mobile ad hoc networks. Ad Hoc Networks, vol. 2 (1), pp: 1-22.

[14]M. Conti, S. Giordano, 2007. Multihop ad hoc networking: The theory. IEEE communications Magazine, vol. 45 (4), pp. 78-86.

[15] M. Krunz, et al., 2004. Transmission power control in wireless ad hoc networks: Challenges, solutions, and open issues. IEEE Networks, vol. 18 (5), pp. 814.

[16]Q. Han, et al., 2011. Link availability predictionbased reliable routing for mobile ad hoc networks. IET Communications, vol. 5 (16), pp. 2291-2300.

[17] R. Dube, et al., 1997. Signal stability-based adaptive routing (SSA) for ad hoc mobile networks. IEEE Personal Communications, vol. 4 (1), pp. 36-45.

[18]R.-S. Chang, S.-J. Leu, 2006. Long-lived path routing with received signal strength for ad hoc networks. In: Proceeding of IEEE Wireless Pervasive Computing.

[19] S. C. Ergen, P. Varaiya, 2005. On multi-hop routing for energy efficiency. IEEE Communication Letters, vol. 9 (10), pp. 880-881.

[20] S. Carson, J. Macker, 1999. Mobile Ad hoc Networking (MANET): Routing Protocol Performance Issues and Evaluation Considerations. IETF RFC2501.txt, pp. 1-12.

[21] S. Lim, et al., 2009. RandomCast: An energyefficient communication scheme for mobile ad hoc networks. IEEE Transactions on Mobile Computing, Vol. 8 (8), pp. 1039-1051.

[22] Ying-Hong Wang, Chih-Feng Chao, 2006. Dynamic backup routes routing protocol for mobile ad hoc networks. Information Sciences, vol. 176 (2), pp. 161-185.

[23] V. Kawadia, P. R. Kumar, 2004. Principles and protocols for power control in wireless ad hoc networks. IEEE Journal on Selected Areas in Communications, vol. 23 (1), pp. 76-88.

[24] W. K. Lai, et al., 2007. Adaptive backup routing for ad-hoc networks. Computer Communications, vol. 30 (2), pp: 453-464.

[25] W.-F. Wang, P.-H. Shih, 2006. Study on an enhanced link-stability based routing scheme for mobile ad hoc networks. In: Proceedings of IEEE Communications Society on Sensor and Ad Hoc Communications and Networks (SECON), vol. 3, pp. 797-802.

[26]X. M. Zhang, et al., 2010. Exploring the dynamic nature of mobile nodes for predicting route lifetime in mobile ad hoc networks. IEEE Transactions on Vehicular Technology, vol. 59 (3), pp. 1567-1572.

[27] A. Jamakovic, S. Uhlig, 2007. On the relationship between the algebraic connectivity and graph's robustness to node and link failures. In: Proceeding of $3^{\text {rd }}$ EURO-NGI Conference on Next Generation Internet Networks, pp. 96-102.

[28]R. Ramannathan, 2001. Making ad hoc networks density adaptive. In: Proceedings of military communications conference, vol. 2, pp. 957-961.

[29] J.-M. Kim, J.-W. Jang, 2006. AODV based energy efficient routing protocol for maximum lifetime in MANET. In: Proceedings of AICT/ICIW, page 77.

[30] S. Khelifa, Z. M. Maaza, 2010. An energy multi-path AODV routing protocol in ad hoc networks. In: proceedings of I/V Communications and Mobile Network (ISVC), pp. 1-4.

[31]L. Bing, et al., 2009. Cross-layer energy-saving AODV routing protocol. Transactions of Tianjin University, vol. 15, pp. 343-349.

[32]B. Zhang, H. T. Mouftah. 2006. Energy-aware ondemand routing protocols for wireless ad hoc networks. Wireless Networks, vol. 12 (4), pp. 481494.

[33] Alvin Valera, et al., 2010. Improving link failure detection and response in IEEE 802.11 wireless ad hoc networks. In: Proceedings of IEEE PIMRC, pp. $1814-1819$.

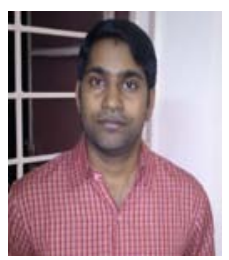

Dhirendra Kumar Sharma is a Ph.D student in Department of Computer Science and Engineering, Indian School of Mines, Dhanbad. Before joining the ISM, he completed his B.Tech degree in Electronics and Communication Engineering from Dr. Ram Manohar Lohia Avadh University, Faizabad UP. His research interest in development of routing protocols for Ad-hoc networks, Wireless sensor networks.

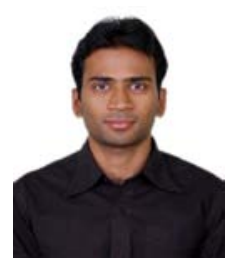

Amar Nath Patra has done masters (M.Tech (CA)) from Indian School of Mines, Dhanbad. He has research interest in Ad Hoc Networks.

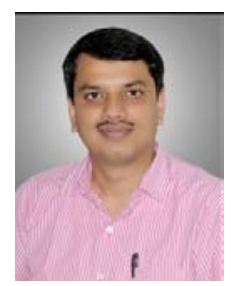

Chiranjeev Kumar is working as an Associate Professor in the Department of Computer Science and Engineering Indian School of Mines, Dhanbad. Before that, he has joined Motilal Nehru National Institute of Technology, Allahabad, UP, in 1998 and worked as Part-Time Lecturer. He was the Gold Medalist of his M.E. batch at MNNIT, Allahabad in year 2001 and obtain the Ph.D. from Allahabad University. He 
has 10 years of teaching and research carrier, he has contributed many research papers in several refereed journals and conference proceedings of National, and International reputes. His main research interests include Mobility Management in Wireless Networks, Ad Hoc Networks, and Software Engineering. He is an IEEE member since 2006. He served as reviewers of many international journals. 